\title{
Motto di spirito e potere eversivo di Chichibio
}

\section{Ada Testaferri}

La quarta novella della VI giornata del Decameron illustra, come le altre dello stesso giorno, la forza del motto di spirito:

Chichibio, cuoco di Currado Gianfigliazzi, con presta parola a sua salute l'ira di Currado volge in riso, e sé campa dalla mala ventura minacciatagli da Currado. (391)

In questa abituale presentazione dell'argomento della novella, Boccaccio coglie alcune delle più importanti caratteristiche del motto di spirito, vale a dire: il suo essere un fenomeno verbale, il suo essere improvviso e, infine, la sua capacità di risolvere una situazione difficile. Questa definizione del motto di spirito, seppure indirettamente dataci dal Boccaccio, non si differenzia troppo dalla teoria freudiana del Witz in generale e del motto di spirito in particolare. Freud parla specialmente di "incongruità" e "soluzione" quali caratteristiche del Witz e queste vengono ancora oggi attribuite al motto di spirito in genere sia dagli odierni psicanalisti che dai linguisti.

Nel presente studio ci proponiamo di analizzare la quarta novella alla luce delle varie teorie concernenti il comico in generale e soprattutto il motto di spirito, visto quest'ultimo come una manifestazione particolare del comico. Il nostro scopo è quello di ottenere una lettura della quarta novella che punti sulla sua specificità di essere una novella-motto e, fatto questo, chiarisca il significato di questa scelta da parte del Boccaccio e la funzione che la novella svolge in seno al Decameron.

Storicamente la ricerca sul comico si è articolata in maniera bipolare e il comico è stato visto o come fenomeno eminentemente psicologico o come fenomeno sostanzialmente artistico. Nel Filebo di Platone si parla di una mescolanza di dolore e piacere come elementi inerenti tanto al tragico quanto al comico, o più propriamente al "ridicolo". Nel dialogo platonico dunque la tendenza è di considerare il riso come prodotto di una situazione psicologica particolare: il soggetto generante il senso del ridicolo, e quindi il riso, provoca tale effetto in altri grazie al suo comportamento verbale o gestuale improntato a una nozione enfiata di sé. Il dislivello tra questo sproporzionato senso di sé e la realtà provoca il riso in chi osserva o ascolta. Aristotele, invece, vede nel comico un fenomeno tipicamente artistico che è propriamente inerente alla commedia; dato che la commedia nello studio dei generi è da lui vista come imitazione di ciò che è "basso", il filosofo definisce il 
comico come "difetto" o "bruttezza" da cui, però, non si genera né dolore, né distruzione. Nella latinità Cicerone riprende sostanzialmente la definizione aristotelica, ma, interessandosi del comico all'interno del discorso epidittico, concentra la sua analisi sul rapporto tra oratore e pubblico, vale a dire tra emittente dell'espressione comica e ricevente. Cicerone raccomanda una certa prudenza; operata infatti una disgiunzione netta tra soggetto e oggetto del riso, Cicerone suggerisce all'oratore di non eccedere e di rispettare i sentimenti degli ascoltatori affinché, invece del riso, non ne provochi l'ira. Implicitamente, quindi, Cicerone fa riferimento alla co-presenza di una possibile carica di ostilità e di aggressività connesse generalmente al manifestarsi sia del comico che del riso. Nell'analisi data da Cicerone è inoltre importante la relazione tra senso di "attesa" e "sorpresa" a cui faranno riferimento anche studi più recenti. Il riso sarebbe infatti generato se, aspettandoci una cosa, ce ne viene detta un'altra. Tra gli antichi ricordiamo infine Quintiliano che riprende Cicerone, ma insiste nell'importanza dell'emittente quale generatore intenzionale del comico. Il comico è visto, sotto l'influenza di Aristotele, visto sostanzialmente come "basso", ma esso è ottenuto, secondo Quintiliano, tramite un effetto di distorsione di ciò che è "alto e nobile", prodotto però intenzionalmente dall'oratore.

Queste definizioni del comico circolavano più o meno liberamente ai tempi del Boccaccio, né grandi progressi vennero fatti in proposito da chi s'interessò al fenomeno del comico nei secoli successivi. Si deve pertanto arrivare a Freud perché l'indirizzo bipolare dato alla speculazione sul comico dai classici si risolva completamente. Infatti Freud è il primo a operare la fusione tra linguaggio umoristico e linguaggio onirico dando così un nuovo indirizzo alla ricerca sul comico. Il suo libro sul Witz rimane, ancora oggi, uno degli studi più completi in materia. Tuttavia un primo legame tra "incongruità" e riso era stato stabilito già agli inizi dell'Ottocento da un critico letterario, William Hazlitt, che coglieva anche un'altra caratteristica tipica del Witz, e cioè la sua "discontinuità" (Suls 40). Più recentemente Arthur Koestler parla di bisociazione improvvisa di un evento mentale con due matrici incompatibili; similmente il linguista Greimas definisce la barzelletta una specie di mini-racconto letterario capace di portare al "livello della coscienza le variazioni delle isotopie del discorso, variazioni che nello stesso tempo si finge di dissimulare mediante la presenza del termine connettivo" (85). Questa presenza bi-isotopica darebbe pertanto luogo a una divaricazione ovvero a una selezione diversa di classemi. Infine anche Cesare Segre parla a sua volta di "rottura apparente, dopo la quale (riso o sorriso compreso) il discorso precedente può essere riannodato" (169). Alla luce di questi pareri, siamo dunque in grado di stabilire che il motto di spirito consiste in una bisociazione logica o verbale che si articola in una dissociazione prima e in una successiva associazione di opposti e che genera, nella maggior parte dei casi, il riso.

Cerchiamo ora di stabilire come questo processo dissociativo-associativo possa manifestarsi attraverso il linguaggio. Alla base di questo processo 
dissociativo-associativo c'è, come abbiamo potuto vedere, la forte tendenza all'ambiguità vista come una delle proprietà inerenti al linguaggio stesso. Shultz e Pilon hanno riscontrato quattro fenomeni di ambiguità: a) di tipo lessicale; b) di tipo fonologico; c) della struttura di superficie; d) della struttura profonda (731). Ma perché si abbia il riso queste ambiguità, insite nel linguaggio stesso, devono essere messe in rilievo da un'intenzionalità cosciente nell'atto di produrle e da una disponibilità, seppure inconscia, a capirle. Ci si chiede pertanto quali possano essere le spinte di carattere psicologico che producono il motto di spirito, giocando appunto su queste possibili ambiguità del linguaggio. Koestler, rifacendosi a Freud, parla di "aggressività e ansietà"; al contrario Nerhard suggerisce che, perché la reazione spiritosa si verifichi, bisogna che il soggetto si senta a suo agio, cioè in un ambiente privo di minacce. La maggior parte degli studi contemporanei accoglie senza riserve la teoria freudiana. A nostro avviso, tuttavia, l'affermazione di Nerhard non è priva d'importanza. Mentre Koestler, come Freud, si concentra soprattutto sullo studio del movente interno al motto di spirito, Nerhard si riferisce specialmente alla sua possibile ricezione; questa viene effettuata in modo positivo dal ricevente solo se, appunto, quest'ultimo si trova a proprio agio per poter ridere o sorridere. Tuttavia nella teoria freudiana del Witz è previsto per prima cosa un certo dosaggio di "aggressività" che, ovviamente, si manifesterà in gradazioni appropriate ai singoli casi e, secondariamente, "il riso" stesso viene visto come momento conciliatore che libera sia dall'ostilità repressa che da altre possibili inibizioni.

Suls ha elaborato un modello cognitivo che ci sembra soddisfacente e che mette in evidenza l'importanza del fattore "sorpresa" come dato praticamente essenziale al verificarsi del motto di spirito (o della battuta spiritosa):

\section{Story set up.}

\section{Prediction.}

3. Is it ending as predicted?

4. $Y E S=$ no surprise, no laughter $/ \mathrm{NO}=$ surprise

5. Find rule that makes ending following from preceding material.

6. Is the rule found?

7. YES $=$ laughter $/$ NO $=$ puzzlement.

La cosiddetta "resa" del motto di spirito o della barzelletta dipenderebbe infatti dal buon dosaggio tra un primo momento, in cui le aspettative del 
ricevente sono in un certo senso frustrate dalla presenza dell'imprevedibile, e un secondo momento, in cui invece il ricevente riesce a riannodare la divergenza isotopica e a risolvere quindi l'ambiguità. Il modello cognitivo di Suls, inoltre, illustra molto chiaramente il verificarsi del fenomeno di divaricazione isotopica di cui parla Greimas e anzi fissa i luoghi della dissociazione e della successiva associazione come momenti ben definiti del processo cognitivo che, tra l'altro, non si effettua sia se manca la dissociazione isotopica (cioè se l'emittente e il ricevente seguono uno stesso canale isotopico), sia se, generato il gioco bi-isotopico, questo non viene percepito come tale dal ricevente, che non sarà quindi in grado di "capire" la battuta.

Infine, essendo il motto di spirito un fenomeno esclusivamente verbale (anche se spesso la comunicazione gestuale può esservi accoppiata), vediamo di studiarlo come possibile fenomeno narrativo. Romana Rutelli propone senz'altro di applicare al Witz le stesse tecniche di studio che modellano la narrazione, in quanto anche le battute spiritose più ridotte, nello studio sul Witz fatto da Freud, sono incorporate in un testo narrativo, cioè è Freud stesso che le racconta. Anche per il Segre l'elemento narrativo è importante ed egli traccia una tipologia strutturale della comunicazione spiritosa comparando la barzelletta all'arguzia o motto di spirito, comparando cioè una forma di humour piuttosto popolare e spesso orale a una forma più elaborata e scritta. Secondo Segre l'arguzia o motto di spirito deve assolutamente essere inglobata nel testo. Questo sarà più ampio nell'arguzia che nella barzelletta: non solo, ma nell'arguzia o nel motto di spirito sarà praticamente impossibile sostituire alcun elemento del testo, cosa invece possibile in molte barzellette. Per la barzelletta, Segre accetta la struttura ternaria proposta da Marfurt (Einleitung, Dramatisierung, Pointe), mentre sostiene che l'arguzia e il motto di spirito presentano invece una struttura monoblocco (Segre 170). Diversamente da Segre, Romana Rutelli propone per il motto di spirito una struttura a nostro avviso più soddisfacente e che si articola in due fasi: narrazione-rappresentazione. Greimas stesso del resto riconosce lo schema bifasico come tratto formale costante del genere storiella o barzelletta (85). Lo schema proposto dalla Rutelli (205) è infatti perfettamente sovrapponibile alla quarta novella della VI giornata del Decameron che, a questo punto del nostro studio, passiamo ad analizzare alla luce delle varie teorie presentate.

Per praticità, suddividiamo la novella in tre scene o sequenze:

1. sequenza iniziale: a) presentazione dei personaggi; b) caccia e cattura della gru; c) cottura della gru; d) Chichibio spicca la coscia e la dà a Brunetta (prima infrazione);

2. sequenza mediana: e) presentazione della gru a tavola; f) domande di Currado; g) risposta di Chichibio (seconda infrazione); h) minaccia di Currado;

3. sequenza finale: i) viaggio e ricerca delle gru; 1) rinvenimento delle gru; m) reazione di Currado; n) motto di spirito (terza infrazione); o) conciliazione. 
Ci concentreremo soprattutto sulla sequenza finale, dato che è in essa che si verifica la risposta spiritosa di Chichibio. Lo schema bifasico è presente come narrazione quando nella novella si descrive la partenza mattutina dei due viaggiatori; tanto è vero che questa sezione contiene a sua volta una drammatizzazione implicita e resa perciò con il discorso indiretto: "e comandò che $\mathrm{i}$ cavalli gli fosser menati; e fatto montar Chichibio sopra un ronzino ..." (392). Si passa poi alla fase della vera e propria rappresentazione con il discorso diretto ("Tosto vedremo chi avrà iersera mentito, o tu o io"), a cui fa seguito una lunga narrazione e quindi, di nuovo, una rappresentazione in forma di discorso diretto: "Assai bene, potete, messer, vedere che iersera vi dissi il vero, che le gru non hanno se non una coscia e un piè, se voi guardate a quelle che colà stanno" (392). D'ora in poi la rappresentazione viene privilegiata, essendo il dialogo, cioè la riproduzione dello scambio orale, la forma necessaria all'inverarsi del motto. A questo punto della novella però sono stati compiuti alcuni passi essenziali alla costruzione e ricezione del motto e vale a dire, riferendosi allo schema cognitivo di Suls, si sono già verificate nel testo la preparazione (Story set up), l'aspettativa di Currado e del lettore (Prediction), cioè che Chichibio verrà smentito alla vista delle gru, e infine la sorpresa, ovvero il ritrovamento di gru a una gamba. Chichibio funge da emittente e Currado da destinatario. La carica aggressiva, descritta da Freud, dovrebbe rivelarsi in questo punto del testo, dato che il motto di spirito si manifesta attraverso un'infrazione o violenza, per lo più di carattere linguistico. Infatti Chichibio sostiene quanto già detto la sera avanti (sequenza mediana: g) e ripete cioè un'infrazione di tipo sia ontologico che linguistico, alterando la definizione propria di "gru".

Freud ci ha suggerito alcuni degli oggetti dell'aggressività: la ragione, il giudizio critico, il sesso e la repressione in genere; egli specifica inoltre nella classificazione dell'umorismo tendenzioso che vi sono delle storielle che egli definisce "scettiche", il cui scopo è non tanto di attaccare una persona o un'istituzione, quanto la sicurezza stessa della nostra conoscenza, una delle nostre proprietà speculative (161). A nostro avviso è proprio il modello del motto di spirito "scettico" quello che caratterizza la novella quarta della VI giornata del Decameron. L'infrazione è presente nella novella in questione, prima nelle azioni (prima infrazione, sequenza iniziale: d), poi nel linguaggio (seconda infrazione, sequenza intermedia: $g$ ). D'ora in poi si stabiliscono due tipi di verità, quella di Currado: gru = bipede, e quella di Chichibio: gru = monopede. L'infrazione di Chichibio è quindi, a questo punto del racconto, un'infrazione di carattere solo ontologico. Si stabilisce con l'oggetto gru un referente plausibile (bipede) e uno impossibile (gru monopede), donde lo scontro tra $\mathrm{i}$ detentori di teorie opposte che richiede una prova: "io il voglio veder domattina e sarò contento" (392). Al mattino successivo il fenomeno bisociativo si verifica non solo nel linguaggio, ma anche nell'esperienza visiva, quindi a livello di conoscenza empirica. Infatti i due uomini vedono, in un primo momento, una gru a una gamba (si ha cioè la 
verifica dell'impossibile), poi, grazie all'intervento di Currado, si stabilisce la plausibilità (gru a due gambe). Segue infine l'operazione di scarto tra le due verità postulate, operata rapidamente da Chichibio che ricorre al motto di spirito. Nel formulare questo, Chichibio segue fedelmente questo schema: associazione-dissociazione-associazione. Esso è reperibile perfettamente nella manifestazione di superficie del linguaggio: "Messer sì" (accettazione) "ma voi non gridaste ho ho a quella di iersera" (negazione) "ché se gridato aveste, ella avrebbe così l'altra coscia e l'altro piè fuor mandata, come hanno fatto queste" (accettazione) (393). Il momento difficile, con la sua carica aggressiva che caratterizza il motto di spirito, specialmente di tipo scettico, è rilevato nella narrazione-descrizione che precede il motto stesso: "Chichibio quasi sbigottito, non sapendo egli stesso donde venisse ... " (393). Tuttavia l'incrocio delle due verità, scontro terrificante, è superato tramite il motto che opera un effetto di humour a carattere terapeutico-conciliante: "A Currado piacque tanto questa risposta che tutta la sua ira si convertì in riso" (393). Nel ricorrere alla risposta spiritosa, Chichibio compie in realtà una terza infrazione (sequenza finale: $n$ ) la cui natura è alquanto complessa. Quest'infrazione è sostanzialmente di carattere logico. Chichibio trasforma in logico un atto illogico, ossia quello di gridare "ho, ho" ad una gru cotta. Sul piano del discorso, questo dislocamento della logica si manifesta con la presenza di pronomi relativi ("quella") e personali ("ella"), di aggettivi indefiniti ("l'altra", "l'altro" e infine "queste") che sono tutti referenti anaforici che collegano il presente al passato ("iersera"), e che mettono in relazione questo testo al co-testo precedente. Anche questa risposta di Chichibio giunge nuovamente inattesa, dando luogo all'effetto di "sorpresa" (vedi sopra), ma, al tempo stesso, essa si riallaccia al co-testo precedente, permettendo sia a Currado che al lettore di scoprire la regola che collega l'evento attuale con il precedente e, sempre seguendo lo schema cognitivo di Suls, generando il riso. Vediamo tuttavia di analizzare ulteriormente la resa di questa risposta: essa si contrappone non solo alla logica ma anche alla sicurezza ontologica di Currado, vale a dire è un motto di spirito "scettico". Non bisogna perdere di vista infatti che la gru riveste un ruolo importante in tutta la novella, dato che essa funge da attante-oggetto e rappresenta un valore desiderabile, tanto è vero che Currado se ne impossessa andando a caccia; essa, inoltre, è "grossa e giovane" (391), quindi buona da figurare in una cena di un certo tenore. La gru è percepita come oggetto-valore anche da Brunetta, la serva, che vuole averne assolutamente un pezzo. Questo valore della gru come bene di scambio viene in effetti stabilito proprio nel dialogo tra Brunetta e Chichibio: "se tu non la mi dai, tu non avrai mai da me cosa che ti piaccia" (391), la cui inferenza sessuale è fin troppo ovvia. In seguito, la gru viene presentata mutila a tavola dove siedono alcuni ospiti forestieri. Currado si meraviglia e si turba alla spiegazione data da Chichibio. La gru, quindi, è anche per Currado un oggetto-valore che si riferisce alla qualità del suo status ("vita cavalleresca tenendo" 391) ed essa, perciò, avrebbe dovuto fargli fare buona figura con gli 
ospiti. Infine anche per Chichibio la gru è un oggetto-valore; esso appartiene apertamente all'ordine socio-economico del denaro e del potere: a) scambio della coscia per la donna; b) furto ai danni del padrone. Tuttavia, nello scontro tra Currado e Chichibio il valore socio-economico rivestito dall'oggetto gru viene oltremodo caricato dall'aggiunta d'un ulteriore valore qualificante: gru a due gambe vs gru a una gamba, e l'asserzione di Chichibio postula una realtà altra e certo non nota a Currado. L'opposizione qualificante aggiunta al lessema gru dà quindi luogo a un sistema di rapporti nuovo che preferiamo rappresentare ricorrendo allo schema del carrè semiotique:

possibilità

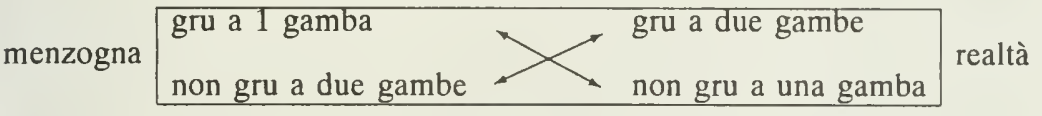

irrealtà

Non è tanto al potere sccio-economico che Chichibio mira (anche se è poi vero che si appropria indebitamente di una coscia della gru), quanto alla rottura d'un sistema ontologico dato e inflessibile, rappresentato nel carrè semiotique dalla relazione d'implicazione di destra (cioè gru a due gambe). A Chichibio preme invece di stabilire una relazione tra contrari (gru a una gamba e anche gru a due gambe), che è infatti rappresentativa d'una visione della realtà in chiave possibilistica.

Muscetta ha fatto riferimento all'influenza, per quanto indiretta, di Occam sul Boccaccio ed anche Eco, parlando del tardo medioevo, segnala in esso la presenza d'una ricerca di moduli d'ordine nuovi, che rappresentino più da vicino la nuova classe mercantile incapace di riconoscersi in quell'ordine universale che aveva impostato concettualmente l'opera di Dante e prodotto l'estetica tomista. Nella quarta novella si assiste proprio a questo rifiuto d'un modello ideologico improntato alla concezione di un ordine universale, vale a dire al fatto che la gru, essendo un uccello, sia unicamente e in tutte le occasioni un bipede. Il portatore della nuova ideologia è Chichibio, descritto nel testo come uno che viene da un altro luogo (è veneziano) e soprattutto come uomo nuovo (è detto infatti "nuovo bergolo"). Currado, invece, è rappresentato come notabile della città e come detentore del potere socio-economico. Come padrone e notabile, infatti, Currado s'impadronisce dell'oggetto-valore gru, tramite l'esercizio signorile della caccia, ma Chichibio trasforma subito questo oggetto, prima cuocendolo (il che gli era permesso), poi presentandolo privo d'una coscia (il che non gli era permesso) e, infine, trasformandolo in un simbolo portatore d'una nuova Weltanschauung, cosa che in un primo momento era sembrata un'assurdità. La risposta di Chichibio in forma di motto di spirito significa dunque molto di più che un semplice espediente tra l'ingenuo e lo spiritoso per far accettare un furto senza confessarlo e chiederne perdono al padrone. L'avvento del nuovo modo di pensare è riflesso nel testo proprio da Currado, che, ridendo, si apre alla nuova concezione della realtà ed accetta con liberalità la risposta di Chichibio. Il potere della parola è certo 
uno dei temi base del Decameron. Tramite il genere della novella-motto il Boccaccio sottolinea questo potere illustrandone l'effetto eversivo, la carica rivoluzionaria capace di rigenerare, proprio grazie all'effetto liberatore-conciliatore del riso, una visione ormai irrigidita della realtà che, pur restando ancorata al suo passato, cerca di aprirsi ai valori nuovi dell'umanesimo imminente, e, soprattutto, alle necessità e al pragmatismo mercantilista della nuova borghesia.

\section{York University}

\section{OPERE CITATE}

Aristotele. Dell'arte poetica. Ed. C. Gavalotti. Milano: Mondadori, 1974.

Boccaccio, Giovanni. Decameron. Ed. C. Segre. Milano: Mursia, 1984.

Cicerone, Marco Tullio. Opere retoriche. Ed. G. Norcio. Torino: Utet, 1976.

Eco, Umberto. Il problema estetico in Tommaso d Aquino. Milano: Fabbri-Bompiani-Sonzogno, 1970.

Fornari, Franco, ed. La comunicazione spiritosa. Il motto di spirito da Freud a oggi. Firenze: Sansoni, 1982.

Freud, Sigmund. Jokes and Their Relation to the Unconscious. Tr. James Slachey. Harmondsworth: Pelican Books, 1983.

Greimas, Algirdas J. Semantica strutturale. Milano: Rizzoli, 1968.

Hazlitt, W. Carew. Lectures on the English Comic Writers. London: Taylor e Hessey, 1819.

Koestler, Arthur. The Act of Creation. New York: Macmillan, 1964.

Marfut, 1. Bernard. Textsorte Witz. Möglichkeiten einer sprachwissenschaftlischen TextsortenBestimmung. Tübingen: Niemeyer, 1977.

Muscetta, Carlo. Giovanni Boccaccio. Bari: Laterza, 1974.

Nehardt, F.K. Goeran. "Operationalization of Incongruity in Humour Research: A Critique and

Suggestions". In Chapman, Tony e Hugh Foot eds. Humour and Laughter: Theory, Research and Applications. London: Wiley, 1976.

Plato. Philebus. Tr. J.C.B. Gosling. Oxford: Clarendon Press, 1975.

Quintiliano, Marco Fabio. L'istituzione oratoria. Ed. R. Faranda e P. Pecchiura. Torino: Utet, 1979.

Rutelli, Romana. "Messa in scena e struttura bifasica del Witz". In Fornari, Franco: 189-214.

Segre, Cesarc. "II Witz e la pragmatica del testo". In Fornari, Franco: 165-88.

Shultz, T.R. and R. Pilon. "Development of the ability to detect linguistic ambiguity." Child Development 44 (1973): 728-33.

Suls, Jerry. "Cognitive Processes in Humour Appreciation”. In Handbook of Humour Research. Eds. Paul E. McGhee e Jeffrey H. Goldstein. 2 vols. New York: Springer-Verlag, 1983: 1.39-57. 